\title{
Best-fit epidemiological models for COVID-19: New cases in Korea
}

\author{
Zixin Yin ${ }^{1,2}$ \\ Haoyi $\mathrm{An}^{1}$ \\ David W. Shanafelt ${ }^{1,3}$
}

\footnotetext{
${ }^{1}$ Berry Institute, Pasadena, California, USA

${ }^{2}$ Dickinson College, Department of Computer Science, Carlisle, Pennsylvania, USA

${ }^{3}$ Université de Lorraine, Université de Strasbourg, AgroParis Tech, Centre national de la Recherche Scientifique (CNRS), Institut National de la Recherche Agronomique (INRA), Bureau d'Économie Théorique et Appliquée (BETA), Nancy, France
}

This paper is the result of a term project for the course PBL Data Science in Ecology with the Berry Institute, completed during the Spring of 2020. It represents a short, independent research project developed and carried out by the students under the supervision and collaboration with the instructor.

Keywords: basic reproduction number; COVID-19; epidemiological models; parameter estimation; Republic of Korea

Word Count: 3094 (excluding references) 


\section{ABSTRACT}

The outbreak of COVID-19 has turned the year 2020 upside-down. While scientists have made progress in our understanding of the structure, transmission, and treatment of the disease, there remains a question as to the long-term immunity of recovered patients. Our research attempts to answer this question by fitting model parameters of three epidemiological models (SIR, SEIR, and SIRS) to COVID-19 data from the Republic of Korea. We find that the SIRS model is the best-fit model, which suggests that - at least for the Republic of Korea during our study period - people obtain limited immunity post-infection.

\section{INTRODUCTION}

The ongoing worldwide pandemic is a highly contagious disease caused by a novel coronavirus pathogen (SARS-CoV-2 or COVID-19). Since its first discovery in December 2019 in Wuhan, China, the coronavirus quickly spread throughout the world, causing over 6 million reported cases and 300 thousand reported deaths around the globe in 6 months (WHO, 2020a). While the symptoms of COVID-19 are usually mild for the general population, the disease can be lethal to at-risk patients such as the elderly and individuals with respiratory or auto-immune deficiencies (WHO, 2020b).

In order to slow the spread of the epidemic, many countries decided to shut down productive activities, implementing social distancing and lockdowns. Unfortunately, practicing lockdowns can have large, negative effects on the world economy and social stability in many countries, including Italy, Spain, and the United States. With the world looking towards the end of the pandemic, it is imperative that scientists have tools to measure the progression of the pandemic, gauge the effectiveness of a disease management policy, predict new cases, and dispense medical resources and social welfare.

A valid question is whether individuals can be re-infected after recovery or if they are immune. Studies have shown that recovered patients have antibodies to the virus in their blood, yet its persistence declines within months of infection and little is understood how the presence of antibodies translates to actual immunity (Ibarrondo et al. 2020; Long et al., 2020). Overall, there is little conclusive evidence that the presence of antibody to COVID 19 can cause long-lasting immunity (WHO, 2020c). We contribute to this question by calibrating several epidemiological models - with and without re-infection - to the number of the new cases of COVID-19 in the Republic of Korea from January $20^{\text {th }}$ to April $30^{\text {th }}, 2020$. We find that the epidemiological model with re-infection provides the best-fit to the data, and according to our analysis, it is likely that post-infection, people are immune for only a limited time. 


\section{METHODS}

\section{Data and disease management in the Republic of Korea}

We calibrate a set of epidemiological models to the number of the new cases of COVID-19 in the Republic of Korea from January $20^{\text {th }}$ to April $30^{\text {th }} 2020$ (Figure 1) (WHO, 2020a). The Republic of Korea was one of the first countries to report cases of COVID-19, and the data exhibits a complete epidemic curve within the first three months of the epidemic.

Unlike many countries, the Republic of Korea managed to curb the pandemic without lockdown. After the first case was reported on Jan $20^{\text {th }}$, the Korean government took early action to prepare large quantities of medical testing kits, conducted widespread testing, isolated infected individuals in government shelters, and implemented social distancing strategies (Kim, 2020; Lee and Lee, 2020). As of April 30 ${ }^{\text {th }}$, the Republic of Korea had 10,765 total cases and 247 total deaths. For comparison, Italy had 203,591 total cases and 27,682 total deaths (WHO, 2020a).

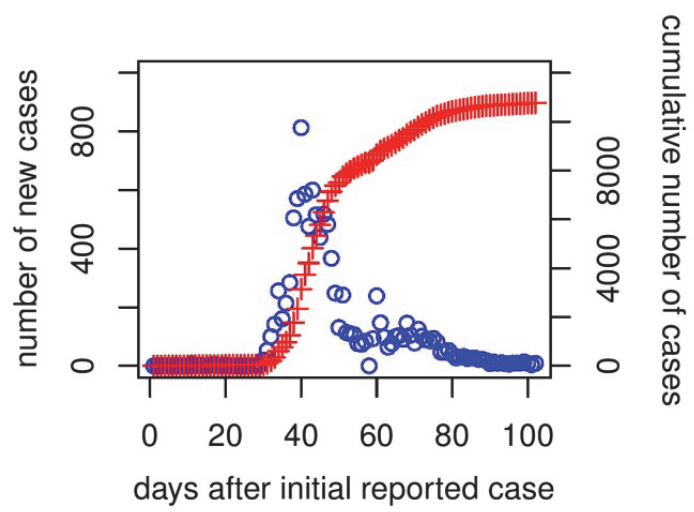

Figure 1. Number of new and cumulative reported cases in the Republic of Korea. Marker color and style indicates the number of new (blue, circle) and cumulative (red, plus) reported cases.

\section{Epidemiological models}

We fit three epidemiological models to the data (Brauer, 2006; Brauer and CastilloChavez, 2012; Hethcote, 2000). Each is a type of compartmental epidemiological model in which the total population, $N$, is divided into classes or types of individuals, where each class represents some state of disease infection ${ }^{1}$.

\footnotetext{
${ }^{1}$ Our compartmental epidemiological models are mechanistic models, which measure the number of individuals of certain disease states within a population. In contrast, phenomenological models such
} 
Our first model is the classic Susceptible-Infected-Recovered or SIR model, which has three classes of individuals: susceptible, $S$ (not infected, but with the potential to become so); infectious, $I$ (who have the disease and can spread it to others); and recovered, $R$ (who have contracted the disease, but have recovered and are immune).

The change in the number of individuals in each class of individuals at time $t$ is given by the equations,

$$
\begin{aligned}
\frac{\mathrm{d} S}{\mathrm{dt}} & =-\beta S(t) \frac{I(t)}{N(t)} \\
\frac{\mathrm{dI}}{\mathrm{dt}} & =\beta S(t) \frac{I(t)}{N(t)}-\gamma I(t) \\
\frac{\mathrm{dR}}{\mathrm{dt}} & =\gamma I(t)
\end{aligned}
$$

where the $N(t)=S(t)+I(t)+R(t)$. The parameter $\beta$ is the infection rate, which tells the probability that infectious and susceptible individuals make contact and transfer the infection. The parameter $\gamma$ is the recovery rate, which tells the percentage of infectious that recover at each moment in time. The inverse of the recovery rate is often interpreted as the "duration" of infection.

The second model builds onto the SIR by adding an additional class of individual: asymptomatically-infectious, $E$. Asymptomatic individuals have been exposed and infected, can infect others, but do not show any outward symptoms of disease. The dynamics of the SEIR model over time can be written as,

$$
\begin{aligned}
\frac{\mathrm{d} S}{\mathrm{dt}} & =-\beta S(t) \frac{E(t)+I(t)}{N(t)} \\
\frac{\mathrm{dE}}{\mathrm{dt}} & =\beta S(t) \frac{E(t)+I(t)}{N(t)}-\alpha E(t) \\
\frac{\mathrm{dI}}{\mathrm{dt}} & =\alpha E(t)-\gamma I(t) \\
\frac{\mathrm{dR}}{\mathrm{dt}} & =\gamma I(t)
\end{aligned}
$$

where $N(t)=S(t)+E(t)+I(t)+R(t)$ and $\alpha$ is the incubation rate, or the rate at which latent, asymptomatic individuals develop symptoms. In contrast to the SIR model, the transmission rate $B$ represents the probability that a susceptible individual contracts the infection after making contact with an asymptomatic or infected individual.

the generalized-growth model (GGMs) or the general Richard's model (GRM) measure the number of new cases per day (Chowell, 2017; Shanafelt et al., 2017). 
The final model allows for recovered individuals to become re-infected. That is, a recovered individual does not have permanent immunity from the disease and eventually becomes re-susceptible to infection. The equations of the SIRS model are written as,

$$
\begin{aligned}
\frac{\mathrm{d} S}{\mathrm{dt}} & =-\beta S(t) \frac{I(t)}{N(t)}+\theta R(t) \\
\frac{\mathrm{dI}}{\mathrm{dt}} & =\beta S(t) \frac{I(t)}{N(t)}-\gamma I(t) \\
\frac{\mathrm{dR}}{\mathrm{dt}} & =\gamma I(t)-\theta R(t)
\end{aligned}
$$

where $N(t)=S(t)+I(t)+R(t)$ as before and $\theta$ represents the proportion of recovered that lose their immunity and return to the susceptible class.

These models neglect births and deaths of individuals, as well as immigration of individuals into the population. Individuals move from one disease class to another, and the total size of the population remains constant for the duration of the epidemic. The validity of this assumption depends on the timescale of the analysis and the nature of the disease in question (Brauer, 2006; Hethcote, 2000). For diseases such as the cold and flu, an epidemic may last weeks or months and accounting for births of new individuals into the population would not be appropriate. For diseases that last a lifetime, such as AIDS/HIV, hepatitis C, or tuberculosis, this may be more reasonable. Our models further assume that the probability of contact between individuals of each disease class are constant and identical, and ignores age structure (Hethcote, 2000) or other disease states such as hospitalized or isolated (Chowell et al., 2003; Riley et al. 2003), or vaccinated (Lenhart and Workman, 2007).

Each of our models was simulated in R version 3.6.3 using a first-order Euler approximation with a step size of 0.25 . All simulations were run for 60 time steps in order to line up with the timescale of the data. Each time step was equivalent to one day in the data. Initial conditions can be found in Table 1. Model parameters were calibrated from the data using the method of sum of squared differences, detailed below.

\section{Fitting models to data: Calibration of model parameters}

We calibrated model parameters using the method of the sum of squared differences (SSD) (Hilborn and Mangel, 1997). The method calculates the squared difference between the epidemiological model output and the empirical data. Because we do not know the "true" number of susceptible individuals, we focus on the number of infectious individuals over time. For our analysis, we fit the number of new infectious individuals over time in the epidemiological model to the new number of cases per day in the data. For the SIR and SIRS models, we fit the number of new symptomatically individuals over time to the data. For the SEIR model, we fit two 
different scenarios: new symptmatically infectious individuals and new asymptomatically-infectious individuals (or new individuals entering the "infected population"). The former assumes that we only observe symptomatic infectious in the data. The latter is more in-line with the practice of perfect, uniform testing: we are able to detect transmission from both asymptomatic and symptomatic individuals to the susceptible population.

To apply the sum of squared differences, we ran a large set of simulations, looping through plausible combinations of parameter values ( $\beta, \gamma, \alpha$, and $\theta$ ). For each individual simulation, we calculated the squared difference or error between the data and its corresponding time points in the model output. The summed value of the squared error for each data point is the SSD. The parameter combination with the smallest SSD yields the set of "best-fit" parameters.

Our approach is a simplified version of that of Chowell et al. (2004, 2007), Chowell (2017), and Citron-Arias et al. (2009). In contrast to studies that place more importance on recent data (Capaldi et al., 2012), we adopt a constant weighting of each data point over time. Similarly, while some studies fix one or more epidemiological parameters and estimate the initial number of infectious (Chowell et al., 2004; 2007), we choose to fix the initial conditions and estimate the epidemiological parameters. Given that the population of residents in the Republic of Korea is just over 51.64 million people and we know modelling the entire country violates the assumption of constant mixing, we focus on a subset of the total population $^{2}$. We set the initial conditions such that the total size of the population is 39,000 individuals $^{3}$, with number of susceptible and infectious making up 99 and 1 percent of the initial population (in SEIR model, the asymtomatic and symptomicallyinfectious classes each compose of 0.5 percent of the population)(Table 1).

\section{RESULTS}

The data best-fit parameters and initial conditions are summarized in Table 1. Illustrations of model outputs for the SIR, SEIR, and SIRS models with the best-fit parameters can be found in Figure 2.

The best-fit parameters for the SIR model are $\beta=0.510$ and $\gamma=0.195$, with a SSD value of $348,023.1$. The best-fit parameters for the SEIR models are $\beta=0.611$, $\gamma=0.011, \quad \alpha=0.106$, with a $\mathrm{SSD}=293,546.8 \quad$ (new symptomatically-infectious individuals) and $\beta=0.460, \gamma=0.232, \alpha=1.00 \quad$ with a SSD $=358,328.4$ (new asymptomatically-infectious individuals). The best-fit parameters for SIRS model are $\beta=0.569, \gamma=0.251, \theta=0.022$, and the SSD value is $253,042.4$. The model with the smallest SSD value is the SIRS model. Hence, our results suggest that, for the

\footnotetext{
${ }^{2}$ World Bank: https://data.worldbank.org/.

${ }^{3}$ The total population size of 39,000 was calibrated using the SIR model. To do so, we found the combination of parameter values and total population size that result in the lowest sum of squared differences.
} 
Table 1. Initial conditions and best-fit parameters.

\begin{tabular}{|c|c|c|c|c|}
\hline \multicolumn{5}{|c|}{ Initial conditions } \\
\hline Model & Susceptible & Asymptomatic & Infected & Recovered \\
\hline SIR & 38610 & - & 390 & 0 \\
\hline SEIR & 38610 & 195 & 195 & 0 \\
\hline SIRS & 38610 & - & 390 & 0 \\
\hline
\end{tabular}

Best-fit parameters

\begin{tabular}{lcccccc} 
Model & $\beta$ & $\gamma$ & $\alpha$ & $\theta$ & $S S D$ & $R_{0}$ \\
\hline SIR & 0.510 & 0.195 & - & - & 348023.1 & 2.62 \\
SEIR $^{*}$ & 0.611 & 0.011 & 0.106 & - & 293546.8 & 61.31 \\
SEIR $^{\dagger}$ & 0.460 & 0.232 & 1.000 & - & 358328.4 & 2.44 \\
SIRS & 0.569 & 0.251 & - & 0.022 & 253042.4 & 2.27
\end{tabular}

${ }^{*}$ Calibrated to the number of new symptomatically infectious individuals over time.

${ }^{\dagger}$ Calibrated to the number of new asymptomatically-infected individuals over time.

Republic of Korea during from January 20th to April 30th, the SIRS model is the best-fit model and it is likely that people recovered from the infection of COVID-19 may not permanently immune to the virus.

Using our best-fit parameters for each model, we can calculate the $\mathrm{R}_{0}$, or basic reproductive number, for each epidemic model. The $\mathrm{R}_{0}$ is the average number of secondary infections produced when a single infected individual is introduced into a population where everyone else is susceptible (Hethcote, 2000). For most simple epidemiological models, if $\mathrm{R}_{0}>1$, the disease will spread through the population; if $\mathrm{R}_{0}<1$, then the disease will die out. Using the standard formulas for calculating $\mathrm{R}_{0}$ for each epidemiological model (Diekmann et al., 1990; Diekmann et al., 2010; Heffernan et al., 2005), the $\mathrm{R}_{0}$ 's for each model are: 2.62 (SIR), 61.31 (SEIR, new symptomatic cases), 2.44 (SEIR, new asymptomatic cases) and 2.27 (SIRS) ${ }^{4}$.

\section{DISCUSSION}

According to our analysis, the SIRS model is the "best" epidemiological description for COVID-19. That is, recovered individuals are not immune for life and eventually become susceptible to re-infection. This is in contrast to the works Fanelli and Piazza (2020), Peng et al. (2020), and Prem et al., (2020), who find that the SEIR model bestfits the data in Italy, China, and France respectively. Indeed, COVID-19 is generally accepted to have an asymptomatic phase (Bi et al., 2020; He et al., 2020; Liet al. 2020). We believe that this discrepancy is due to the Republic of Korea's rapid management response. Widespread testing could negate the effects of asymptomatic

\footnotetext{
${ }^{4}$ Specifically, for the SIR and SIRS models, the $\mathrm{R}_{0}$ is given as $\mathrm{R}_{0}=\beta / \gamma$. For the SEIR, the $\mathrm{R}_{0}$ is defined as $\mathrm{R}_{0}=\beta((1 / \alpha)+(1 / \gamma))$.
} 
spread, which at the extreme could collapse the asymptomatic compartment to approximate an SIR model. This is indeed the case when fitting the SEIR model to the number of new asymptomatic cases (Table 1). As of June 30th, over 1.27 million tests had been administered, which, in combination with social distancing and other hygienic measures (Kim, 2020; Lee and Lee, 2020), will help to minimize asymptomatic spread $^{5}$.
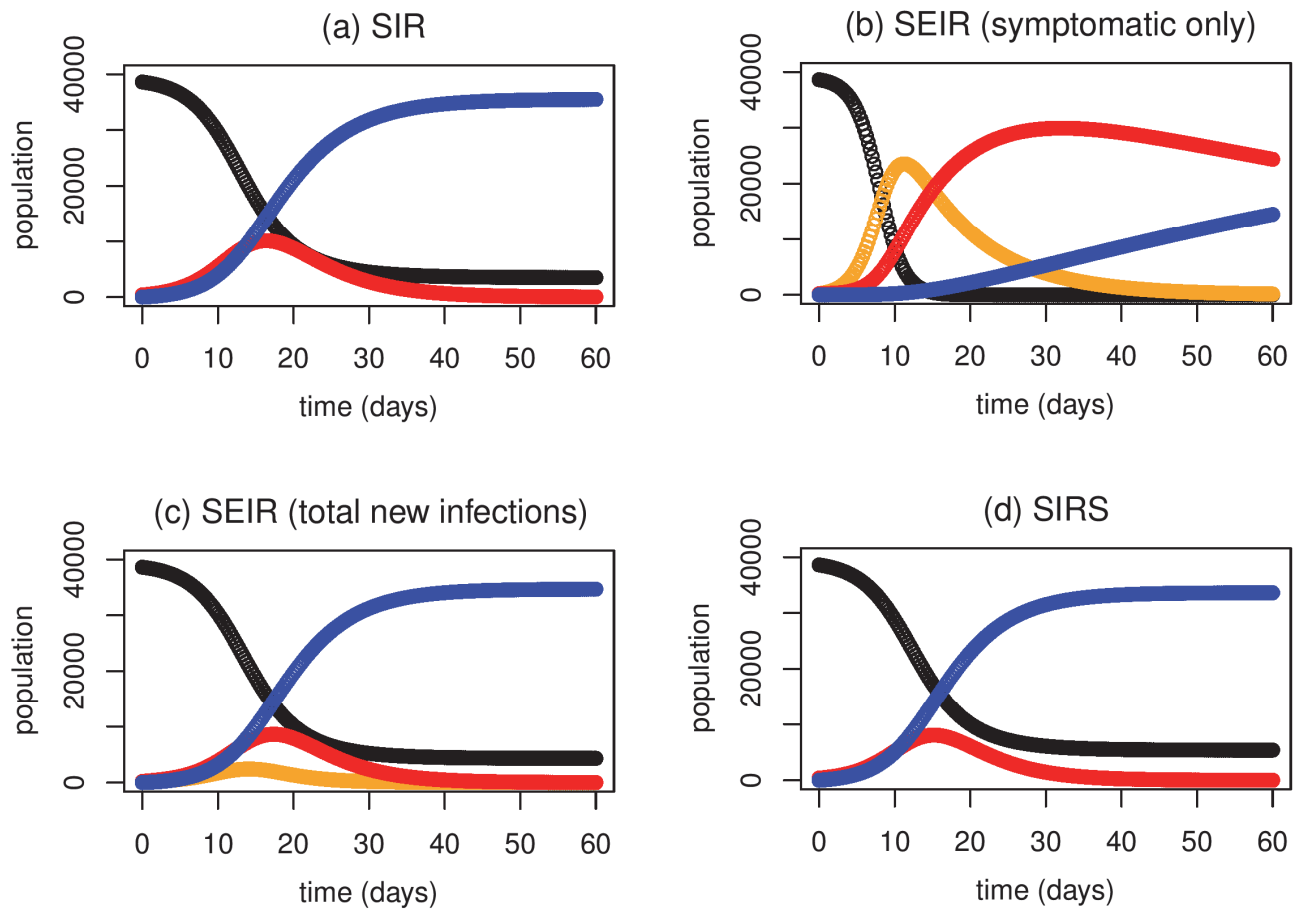

Figure 2. Model dynamics (a-d). All results are using the best-fit parameters for each model (see Table 1 for details). Color indicates the epidemiological compartment: susceptible (black), asymptomatically infectious (orange), symptomatically infectious (red), and recovered (blue). In (b), the model is calibrated using the number of new symptomatically infectious individuals over time. In (c), the model is calibrated to the total number of new infectious individuals.

Our parameter values are in general agreement with the clinical estimates for COVID-19 and SARS. For COVID-19, the median recovery time is 2 weeks for noncritical patients, and 3-6 weeks for patients with severe or critical symptoms (WHOChina Joint Mission, 2020). This translates to $0.024<\gamma<0.071$. For SARS, Chowell et al. (2003) and Chowell et al. (2004) reported transmission rates of 0.75 and 0.25 respectively. Riley et al. (2003) found that the average recovery time was 5-6 days,

\footnotetext{
${ }^{5}$ Statista: https://www.statista.com/.
} 
which translates to $0.167<\gamma<0.20$. Donneley et al. (2003) estimated the average infectious period of 28.4 days or $\gamma=0.035$.

Similarly, estimates of $\mathrm{R}_{0}$ for COVID-19 range from 1.4 to 6.5 , depending on the model and data (Cheng and Shan, 2020) ${ }^{6}$. While the SIR, SEIR (fit to asymptomatically-infectious individuals), and SIRS models fall within this range, the SEIR fit to symptomatically-infectious individuals produces a gross overestimate of $\mathrm{R}_{0}$. This discrepancy in the severity of the epidemics can be observed in the model dynamics (Figure 2), where the SEIR model fit to symptomatically-infectious individuals has by no means converged to its long-term equilibrium within the 60 day time period, and there remains a large number of infected individuals in the population. Our two SEIR scenarios provide potential perspectives of the underlying disease dynamics given assumptions about what we can observe in the data. If we only observe symptomatic cases, then the actual number of individuals who have been infected (symptomatically or not) follows much more severe trajectory than if we can observe all transitions of susceptible individuals into the "infectious population". The actual is likely between these two extremes.

Our model is not without its limitations. First, the model does not account for disease management such as quarantines, vaccinations or treatments for the disease, or social distancing. Implementation of said measures would at the very least affect model parameters, which are assumed constant over time. For example, treatments would increase the recovery rate, while quarantines or social distancing would decrease the transmission rate. More fundamentally, one could incorporate additional compartments in the SIR framework to account for hospitalizations, vaccinations, etc. (Brauer, 2006; Chowell et al., 2003, 2004; Hethcote, 2000). Nor does it account for age structure or high/low risk patients groups, which can matter in COVID-19 transmission and mortality (Wang et al., 2020).

Second, our model is a simple, compartmental epidemiological model with a number of assumptions. Take, for example, the assumption of a well-mixed population. We know that in the reality, people typically have a relatively fixed social circle and residential area, with some people being extroverted while others are insular. Individuals can be reactive, self-isolating (risk averse) or throw COVID-19 parties (risk seeking). Accounting for heterogeneity in contact rates and the contact network can have significant impacts on the overall spread of an epidemic (Ancel Meyers et al., 2005; Brauer, 2006; Morin et al., 2013).

Third, our method of model fitting makes an assumption regarding the quality of information that we observe. The simulation data represents the "true" number of susceptible, infected, and recovered individuals in the population. By fitting the empirical data to the simulation data, we are implicitly assuming that the empirical data reflects the actual number of newly infected individuals. In reality, we possess imperfect information regarding the spread of the epidemic, where - given the nature

\footnotetext{
${ }^{6}$ For comparison, the $\mathrm{R}_{0}$ for $\mathrm{H} 1 \mathrm{~N} 1$ avian influenza was estimated to be 3.4 (Chang, 2010), 1.5-1.9 for Ebola (Khan, 2015), and between 3.5-6 for smallpox (Gani, 2001; Hethcote, 2000).
} 


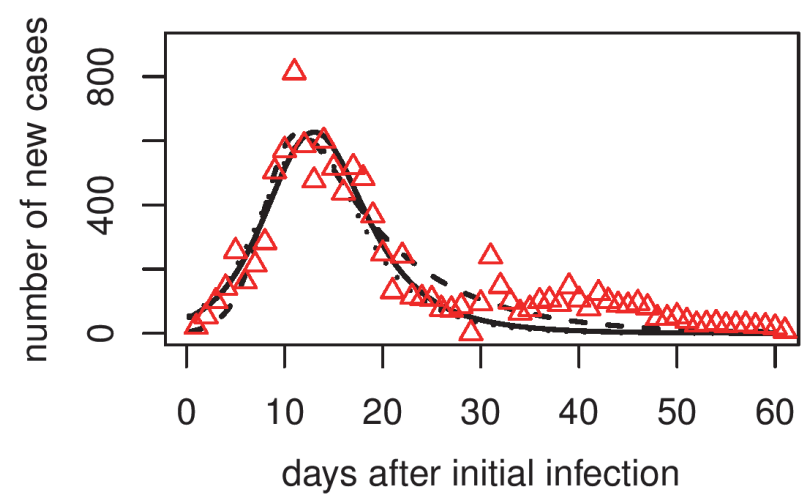

Figure 3. Model fit with Republic of Korea empirical data. Line style corresponds to the symptomatically infectious curves from (a-c): SIR (solid), SEIR (new symptomatically infectious only) (dashed), SIRS (dotted). The empirical data is given by the red triangles.

of the coronavirus - the data is likely an under-representation of the actual number of infections.

Finally, while we hope that our findings are generalizable to other countries, the direct applicability of our results is limited to the Republic of Korea. Indeed, the choice and timing of management strategies varied across the world, which directly affects the disease profile (Figure 1) and the best-fit parameters. Take, for example, the United States, which was much less prepared for the pandemic, experiencing shortages of testing kits and personal protective equipment (WHO, 2020d). While government-mandated lockdowns were able to "flatten the curve", these were implemented on a state-by-state basis and, once lifted, the number of cases have increased exponentially (WHO, 2020a). We leave a deeper investigation of the role of disease management and the epidemic profiles to future work.

\section{REFERENCES}

Ancel Meyers, L., Pourbohloul, B., Newman, M.E.J., Skowronski, D.M., Brunham, R.C. 2005. Network theory and SARS: Predicting outbreak diversity. Journal of Theoretical Biology. 232:71-81.

Bi, Q., Wu, Y., Mei, S., Ye, C., Zou, X., Zhang, Z., Liu, X., Wei, L., Truelove, S.A., Zhang, T., Gao, W., Cheng, C., Tang, X., Wu, X., Wu, Y., Sun, B., Huang, S., Sun, Y., Zhang, J., Ma, T., Lessler, J., Feng, T. 2020. Epidemiology and transmission of COVID-19 in 391 cases and 1286 of their close contacts in Shenzhen, China: A retrospective cohort study. The Lancet Infectious Diseases. 20(8):911-919. 
Brauer, F. 2006. Some simple epidemic models. Mathematical Biosciences and Engineering. 3(1):1-15.

Brauer, F., Castillo-Chavez, C. 2012. Mathematical models in population biology and epidemiology. New York: Springer-Verlag.

Capaldi, A., Behrend, S., Berman, B., Smith, J., Wright, J., Lloyd, A.L. 2012. Parameter estimation and uncertainty quantification for an epidemic model. Mathematical Biosciences and Engineering. 9(3):553-576.

Chang, C.-Y., Cao, C.-X., Wang, Q., Chen, Y., Cao, Z.-D., Zhang, H., Dong, L., Zhao, J., Xu, M., Gao, M.-X., Zhong, S.-B., He, Q.-S., Wang, J.-F., Li, X.-W.2010. The novel H1N1 Influenza A global airline transmission and early warning without travel containments. Chinese Science Bulletin. 55(26):3030-3036.

Cheng, Z., Shan, J. 2020. 2019 Novel coronavirus: Where we are and what we know. Infection. 48:155-163.

Chowell, G. 2017. Fitting dynamics models to epidemic outbreaks with quantified uncertainty: A primer for parameter uncertainty, identifiability, and forecasts. Infectious Disease Modelling. 2:379-398.

Chowell, G., Ammon, C.E., Hengartner, N.W., Hyman, J.M. 2004. Estimating the reproduction number from the initial phase of the Spanish flu pandemic waves in Geneva, Switzerland. Mathematical Biosciences and Engineering. 4(3):457-470.

Chowell, G., Fenimore, P.W., Castillo-Garsow, M.A., Castillo-Chavez, C. 2003. SARS outbreaks in Ontario, Hong Kong, and Singapore: The role of diagnosis and isolation as a control mechanism. Journal of Theoretical Biology. 224:1-8.

Chowell, G., Castillo-Chavez, C., Fenimore, P.W., Kribs-Zaleta, C.M., Arriola, L., Hyman, J.M. 2004. Model parameters and outbreak control for SARS. Emerging Infectious Diseases. 10(7):1258-1263.

Cintron-Arias, A., Castillo-Chavez, C., Bettencourt, L.M.A., Lloyd, A.L., Banks, H.T. 2009. The estimation of the effective reproductive number from disease outbreak data. Mathematical Biosciences and Engineering. 6(2):261-282.

Diekmann, O., Heesterbeek, J.A.P., Metz, J.A.J. 1990. On the definition and the computation of the basic reproduction ratio $\mathrm{R}_{0}$ in models for infectious diseases in heterogeneous populations. Journal of Mathematical Biology. 28:365-382. 
Diekmann, O., Heesterbeek, J.A.P., Roberts, M.G. 2010. The construction of nextgeneration matrices for compartmental epidemic models. Journal of the Royal Society Interface. 7:873-885.

Donnelly, C.A., Ghani, A.C., Leung, G.M., Hedley, A.J., Fraser, C., Riley, S., AbuRaddad, L.J., Ho, L.-M., Thach, T.-Q., Chau, P., Chan, K.-P., Lam, T.-H., Tse, L.-Y., Tsang, T., Liu, S.-H., Kong, J.H.B., Lau, E.M.C., Ferguson, N.M., Anderson, R.M. 2003. Epidemiological determinants of spread of causal agent of severe acute respiratory syndrome in Hong Kong. The Lancet. 361:1761-1766.

Fanelli, D., Piazza, F. 2020. Analysis and forecast of COVID-19 spreading in China, Italy and France. Chaos, Solitons, and Fractals. 134:109761.

Gani, R., Leach, S. Transmission potential of smallpox in contemporary populations. Nature. 414:748-751.

He, D., Zhao, S., Lin, Q., Zhuang, Z., Cao, Z., Wang, M.H., Yang, L. 2020. The relative transmissibility of asymptomatic COVID-19 infections among close contacts. International Journal of Infectious Diseases. 94:145-147.

Heffernan, J.M., Smith, R.J., Wahl, L.M. 2005. Perspectives on the basic reproductive ratio. Journal of the Royal Society Interface. 2:281-293.

Hethcote, H.W. 2000. The mathematics of infectious disease. SIAM Review. 42(4):599-653.

Ibarrondo, F.J., Fulcher, J.A., Goodman-Meza, D., Elliott, J., Hofmann, C., Hausner, M.A., Ferbas, K.G., Tobin, N.H., Aldrovandi, G.M., Yang, O.O. 2020. Rapid decay of anti-SARS-CoV-2 antibodies in persons with mild Covid-19. New England Journal of Medicine. 21 July 2020.

Khan, A., Naveed, M., Dur-E-Ahmad, M., Imran, M. 2015. Estimating the basic reproductive ratio for the Ebola outbreak in Liberia and Sierra Leone. Infectious diseases of poverty. $4: 13$.

Kim, P.S. 2020. South Korea's fast response to coronavirus disease: Implications on public policy and public management theory. Public Management Review. Online Early.

Lee, D., Lee, J. 2020. Testing on the move: South Korea's rapid response to the COVID-19 pandemic. Transportation Research Interdisciplinary Perspectives. 5:100111. 
Lenhart, S., Workman, J.T. 2007. Optimal control applied to biological models. London: Chapman and Hall.

Li, H., Liu, S.-M., Yu, X.-H., Tang, S.-L., Tang, C.-K. 2020. Coronavirus disease 2019 (COVID-19): Current status and future perspectives. International Journal of Antimicrobial Agents. 55:105951.

Long, Q.-X., Tang, X.-J., Shi, Q.-L., Li, Q., Deng, H.-J., Yuan, J., Hu, J.-L., Xu, W., Zhang, Y., Lv, F.-J., Su, K., Zhang, F., Gong, J., Wu, B., Liu, X.-M., Li, J.-J., Qiu, J.F., Chen, J., Huang, A.-L. 2020. Clinical and immunological assessment of asymptomatic SARS-CoV-2 infections. Nature Medicine. 18 June 2020.

Morin, B.R., Fenichel, E.P., Castillo-Chavez, C. 2013. SIR dynamics with economically driven contact rates. Natural Resource Modelling. 26(4):505-525.

Prem, K., Liu, Y., Russell, Y., Kucharski, A., Eggo, R., Davies, N. 2020. The effect of control strategies to reduce social mixing on outcomes of the COVID-19 epidemic in Wuhan, China: A modelling study. The Lancet Public Health. 5(5):e261-e270.

Peng,L., Yang,W., Zhang, D., Zhuge, C. \& Hong, L. 2020. Epidemic analysis of COVID-19 in China by dynamical modeling. ArXiv. PrePrint.

Riley, S., Fraser, C., Donnelly, C.A., Ghani, A.C., Abu-Raddad, L.J., Hedley, A.J., Leung, G.M., Ho, L.-M., Lam, T.-H., Thach, T.Q., Chau, P., Chan, K.-P., Lam, T.-H., Thach, T.Q., Chau, P., Chan, K.-P., Lo, S.-V., Leung, P.-Y., Tsang, T., Ho, W., Lee, K.-H., Lau, E.M.C., Ferguson, N.M., Anderson, R.M. 2003. Transmission dynamics of the etiological agent of SARS in Hong Kong: Impact of public health interventions. Science. 300:1961-1966.

Shanafelt, D.W., Jones, G., Perrings, C., Chowell, G. 2018. Forecasting the 2001 foot-and-mouth disease epidemic in the United Kingdom. EcoHealth. 15(2): 338347.

Wang, D., Hu, B., Chang, H., Zhu, F., Liu, X., Zhang, J., Wang, B., Xiang, H., Cheng, Z., Xiong, Y., Zhao, Y., Li, Y., Wang, X., Peng, Z. 2020. Clinical characteristics of 138 hospitalized patients with 2019 novel Coronavirus-infected pneumonia in Wuhan, China. JAMA. 323(11):1061-1069.

World Health Organization (WHO). 2020a. Covid-19 situation report. Retrieved from: https://www.who.int/emergencies/diseases/novel-coronavirus-2019/situationreports/.

World Health Organization (WHO). 2020b. Coronavirus Overview. Retrieved from: https://www.who.int/health-topics/coronavirus. 
World Health Organization (WHO). 2020c. "Immunity passports" in the context of COVID-19. Scientific Brief. 24 April 2020.

World Health Organization (WHO). 2020d. Shortage of personal protective equipment endangering health workers worldwide. News release. 3 March 2020.

World Health Organization (WHO)-China Joint Mission. 2020. Report of the WHOChina Joint Mission on Coronavirus Disease 2019 (COVID-19). 16-24 February 2020. 